\title{
Maximal Resolution from the Ronchigram: Human vs. Deep Learning
}

Noah Schnitzer ${ }^{1}$, Suk Hyun Sung ${ }^{1}$, Robert Hovden ${ }^{1,2}$

${ }^{1}$ Department of Materials Science and Engineering, University of Michigan, Ann Arbor, MI 48019 USA

2.Applied Physics Program, University of Michigan, Ann Arbor, MI 48109, USA

Real-space atomic-resolution imaging with aberration-corrected scanning transmission electron microscopy (STEM) has revolutionized material characterization. Maximizing resolution, contrast, and total usable beam current is critically dependent on the ability to determine the largest convergence angle (objective aperture) permitted by lens aberrations. However, this quantity is difficult to accurately measure in real charged particle optical systems. For STEM, the electron Ronchigram encodes the optimal convergence angle, but is challenging to quantifiably assess and currently is most accurately evaluated with human expertise [1].

Here we present a deep regression framework which predicts the optimal convergence angle for maximal probe quality and imaging resolution from a simulated Ronchigram. Our neural network operates 500 times faster with 6 times less error than trained microscopists and outperforms common heuristic algorithms that are unrealistically given perfect knowledge of the aberration function.

The widely accepted criteria for selecting a convergence angle for STEM imaging is Rayleigh's quarterwave rule, which promises good image quality for less than a quarter-wave of primary spherical aberration $(\chi<\pi / 4)[2]$. However, this neglects not only non-spherically symmetric astigmatism and coma, but also the parasitic higher-order aberrations arising from corrector lenses. A more robust evaluation of probe quality can be found in the Strehl ratio, which compares the peak intensity of an aberrated probe (Fig. 1a, right) to one without aberrations (Fig 1a, left), $S=\left|\psi_{\chi}(0)\right|^{2} /\left|\psi_{\chi=0}(0)\right|^{2}$ [3]. Notably, this quantity is easy to calculate given a known aberration function, and for spherically symmetric aberrations a Strehl ratio of 0.8 is equivalent to Rayleigh's quarter-wave rule [3]. We show that setting a 0.8 Strehl ratio cutoff estimates the minimum probe size with greater stability and accuracy across a broad distribution of aberrated probes than the two common quarter-wave based heuristics: a quarter-wavelength cutoff for individual aberrations or a quarter-wavelength cutoff for the total aberration function (Fig 1b).

Using a deep convolutional neural network $(\mathrm{CNN})$ trained to estimate convergence angles corresponding to a 0.8 Strehl ratio, we are able to determine the optimal convergence angle with considerably greater accuracy and speed than microscopists (Fig. 1c-d, e-g). Across 675 simulated Ronchigrams with convergence angles ranging from 10 to $70 \mathrm{mrad}$, the network was able to achieve a probe more than $12 \%$ $(6 \mathrm{pm})$ smaller in $0.2 \%$ of the time ( 7 milliseconds per Ronchigram) on average. The network architecture, activation maps of the first and last convolutional layers, and dependence of training on batch size are shown in Figure 2. Training with 98,000 simulated Ronchigrams with semi-random aberrations converged after $\sim 10$ epochs with no signs of overfitting. Beyond convergence angle identification for maximizing resolution, our $\mathrm{CNN}$ approach can provide the rapid feedback required to enable fully automated aberration correction.

\section{References:}

[1] Kirkland, E. J. Ultramicroscopy, 111(11) (2011). p. 1523-1530.

[2] Wetherell, W. B. Applied Optics and Optical Engineering, 8(6) (1980). p. 197.

[3] Mahajan, V. N. JOSA, 72(9) (1982), p. 1258-1266. 


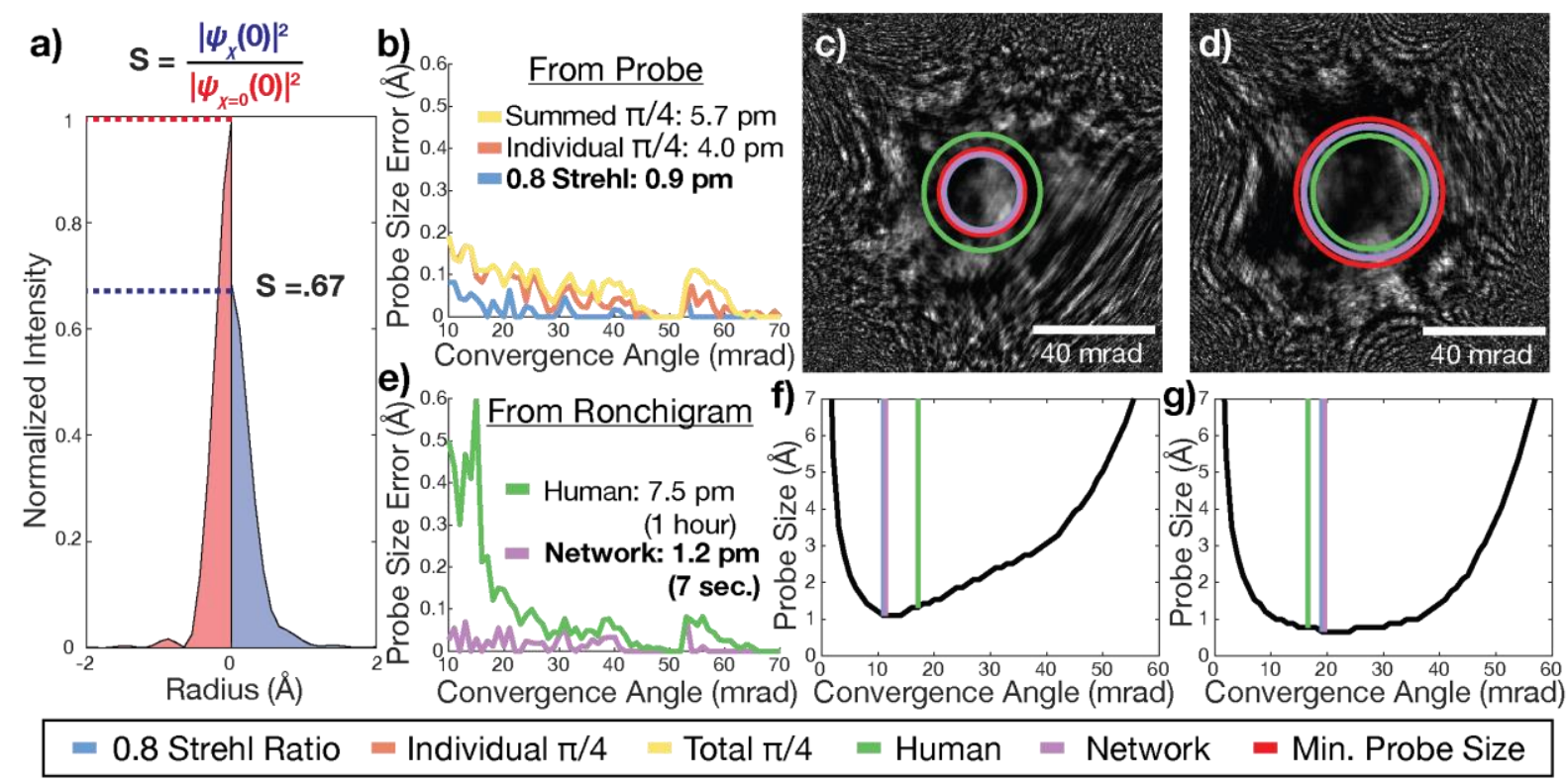

Figure 1 | Maximizing resolution with human vs machine convergence angle estimation. a) The Strehl ratio measures probe quality through comparison of the peak intensity for an aberrated probe (left) and an aberration free probe (right). b) Testing the three probe quality heuristics given perfect knowledge of 675 aberration functions, the 0.8 Strehl ratio most effectively optimizes probe size compared to the global minimum. e) From direct comparison, the CNN strongly outperforms the assessment of multiple trained microscopists. c-d,f-g) Ronchigrams and corresponding probe size dependence on convergence angle, with human, CNN, and Strehl convergence angle selections indicated.

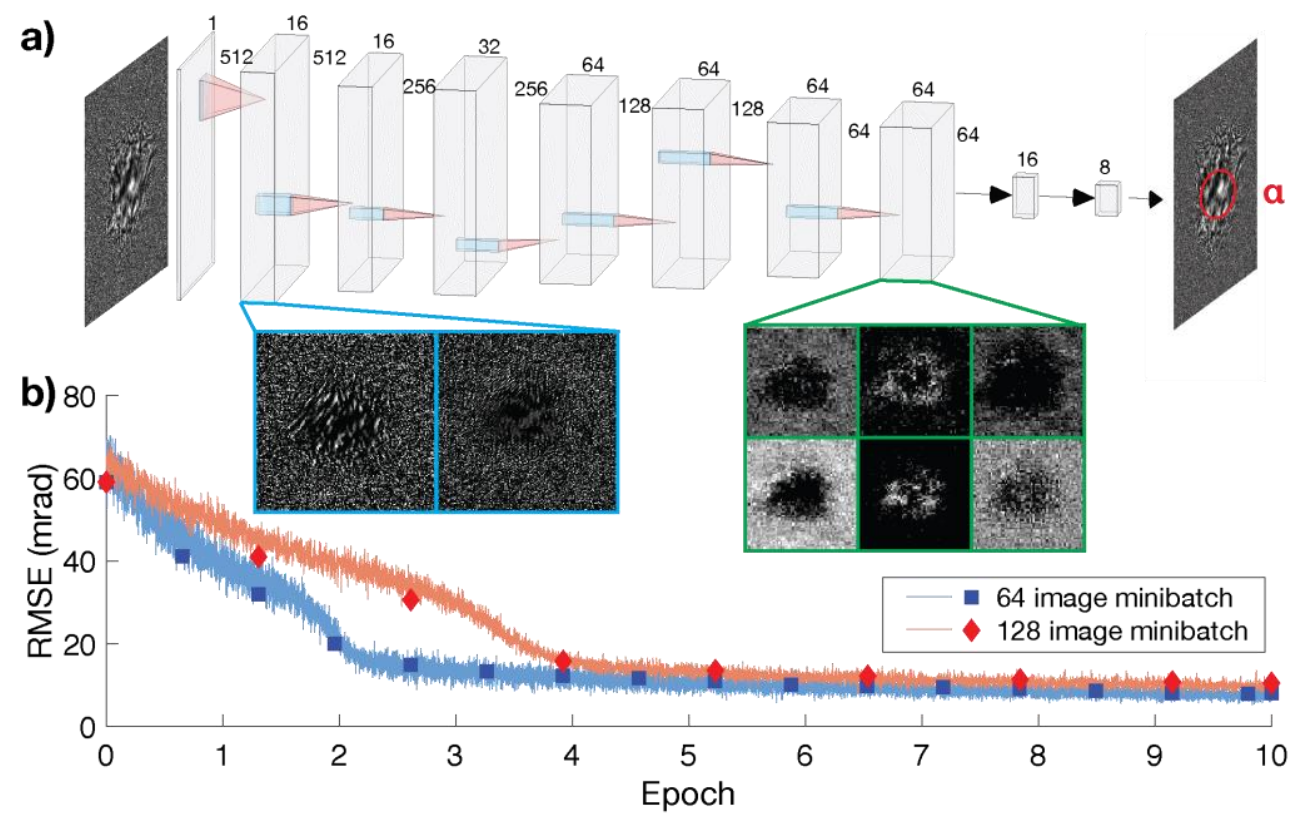

Figure 2 Architecture of convergence angle estimation network. a) Architecture of the CNN, including layer dimensions and filters, along with selected activation maps of the first and last convolutional layers. b) Network error in convergence angle estimation across 10 epochs of training for two different minibatch sizes, as well as performance on a withheld validation set. As expected, the minibatch size affects convergence rate, but does not significantly affect converged error rate. 